TITLE:

\title{
Physicochemical impedance modeling of solid oxide fuel cell anode as an alternative tool for equivalent circuit fitting
}

\section{AUTHOR(S):}

Kishimoto, Masashi; Onaka, Hironori; Iwai, Hiroshi; Saito, Motohiro; Yoshida, Hideo

\section{CITATION:}

Kishimoto, Masashi ...[et al]. Physicochemical impedance modeling of solid oxide fuel cell anode as an alternative tool for equivalent circuit fitting. Journal of Power Sources 2019, 431: 153-161

ISSUE DATE:

2019-08-15

URL:

http://hdl.handle.net/2433/241710

\section{RIGHT:}

(c) 2019. This manuscript version is made available under the CC-BY-NC-ND 4.0 license

http://creativecommons.org/licenses/by-nc-nd/4.0/.; The full-text file will be made open to the public on 15 August 2021 in accordance with publisher's 'Terms and Conditions for Self-Archiving'.; This is not the published version. Please cite only the published version.; この論文は出版社版でありません。引用の際には出版社版をご磪認ご利用ください。 
Physicochemical impedance modeling of solid oxide fuel cell anode as an alternative tool for equivalent

circuit fitting

*Masashi Kishimoto, Hironori Onaka, Hiroshi Iwai, Motohiro Saito, Hideo Yoshida

Department of Aeronautics and Astronautics, Kyoto University, Kyoto 615-8540, Japan

Tel/Fax: +81-75-383-3652

E-mail: kishimoto.masashi.3m@kyoto-u.ac.jp 


\section{Abstract}

A simple but physicochemically meaningful numerical model of a solid oxide fuel cell (SOFC) anode is developed as a tool to understand and reproduce its impedance spectra. The model considers conservations of electrons, ions and gas species within the anode, which are coupled by a non-linear electrochemical reaction model. The developed model is first calibrated by impedance spectra that are experimentally obtained at the open circuit conditions at several temperatures, by adjusting the ionic conductivity, exchange current density and electrostatic capacitance. All of these parameters are confirmed to be within an acceptable range of values found in literature. Also, real anode microstructural parameters obtained by three-dimensional FIB-SEM imaging is implemented to eliminate any other arbitrary fitting parameters from the model. Then, the model is used to reproduce impedance spectra obtained from another anode with different microstructure and also those measured at polarized conditions and at different temperatures, and gives good agreement with the experimental results. Considering the computational resources required to reproduce impedance spectra, the developed model is expected to be used as an alternative to the conventional equivalent circuit fitting.

Keywords: SOFC; impedance; modeling; anode; microstructure 


\section{Introduction}

Solid oxide fuel cells (SOFCs) are considered as promising technology for high-temperature electrochemical energy conversion owing to high efficiency and emission-free, and their commercialization has already started in several countries [1,2]. However, improvement of energy conversion efficiency and cost reduction of the SOFC systems is still of major concern for the larger-scale introduction of the systems to realize the hydrogen society.

In the research and development of SOFC systems, the electrochemical impedance spectroscopy (EIS) is one of the essential techniques to understand the electrochemical behavior of the SOFC electrodes, cells and stacks [3]. The EIS is also useful to monitor performance during durability tests to detect possible degradation phenomena within SOFC systems. During the EIS measurements, a small amplitude of AC current (voltage) with various frequencies is applied to a cell/stack, and the voltage (current) response is recorded to obtain complex impedance for each frequency. The measured impedance typically consists of multiple semi-circles on the Cole-Cole plot, and the number of semi-circles corresponds to that of physicochemical processes in the cell/stack, such as conduction, charge-transfer, gas diffusion and gas conversion. The physicochemical interpretation of the semi-circles has been one of the important research topics in the SOFC research.

For a quantitative understanding of the impedance spectra, equivalent circuit fitting is commonly used. Various types of equivalent circuits have been proposed for SOFC cells, and most of them consist of a combination of resistor-conductor parallel circuits and/or resistor-CPE (constant-phase element) parallel circuits; each parallel circuit can reasonably reproduce one semi-circle in the impedance spectra. Also, a single 
resistor element and an inductor element are also used to represent the bulk conduction resistance and the parasitic inductance due to cell stacking and wiring, respectively. The equivalent circuit fitting is certainly a convenient technique that gives quantitative understandings of the SOFC performance, however, the equivalent circuit models consisting of R-C and R-CPE elements are liable to be used without detailed understandings of the physicochemical processes in the cells.

On the other hand, there are alternative equivalent circuit models that are more rigorously based on physicochemical processes in the cells, such as the transmission line model (TLM) [4, 5], the Gerischer element [6] and the Warburg element. In the transmission line model and the Gerischer element, each of the electron and ion conduction network in an electrode is represented by serially connected resistor elements, and these networks are connected by several R-C or R-CPE parallel circuits, which represent the charge-transfer processes between the electron conduction phase and the ion conduction phase in the electrode. The analytical solution of such circuit gives a complex impedance expression. The Warburg element considers the gas diffusion within the pore phase in an electrode, and the complex impedance expression can be obtained by analytically solving the diffusion equation with an assumption of either finite or infinite diffusion length in the electrode.

Several works reported reasonable fitting of the electrode impedance to the TLM or Gerischer element. However, it has to be emphasized that they assume constant and homogeneous distribution of the electrochemical properties, such as the electronic conductivity, the ionic conductivity and the charge-transfer resistance, in the entire electrode volume [7-11], which is not always the case when the electrode is at polarized conditions. In particular, the charge-transfer resistance is non-linearly dependent on the activation overpotential, 
and hence considered to have non-uniform distribution within the porous electrodes $[12,13]$. Typically, the electrochemical reaction takes place in the vicinity of the electrode-electrolyte interface, therefore the activation overpotential is higher in the region, which forms inhomogeneous distribution of the activation overpotential, and hence that of the charge-transfer resistance. Also, when the temperature is non-uniform within the cell, which often occurs when a reforming process of hydrocarbon or ammonia fuels takes place in the anode, the electronic and ionic conductivities can also have inhomogeneous distribution within the anode. In those situations, the assumptions that are used to derive the TLM and Gerischer elements are no longer valid.

In this study, therefore, an unsteady-state one-dimensional (1D) numerical model is developed to reproduce impedance spectra of SOFC anodes not only at the open circuit conditions but also at the polarized conditions, by directly solving the conservation equations of charge carriers and gas species, which are coupled by the non-linear electrochemical reaction model. Also, the electrode microstructure is taken into account in the model so that the developed numerical model can reproduce impedance spectra obtained from other anodes with different microstructures. Particular attention is also paid to the computational resources required to reproduce impedance spectra so that the developed model can be used as an alternative to the conventional equivalent fitting approaches.

2 Modeling

2.1 Calculation domain and assumptions

In this study, an unsteady-state 1D numerical model was developed for Ni-YSZ (yttria-stabilized 
zirconia) anodes of SOFCs to reproduce their impedance response. The anode microstructure was considered homogeneous and represented by microstructural parameters, such as volume fraction, tortuosity factor and TPB (triple-phase boundary) density. The conservation equations of electrons, ions and gas species were considered and coupled by the electrochemical reaction model as schematically shown in Fig. 1. Although this model appears to be equivalent to the model used to derive the TLM, it should be noted that the charge-transfer resistance $r_{c t}$ is allowed to vary with the local activation overpotential. Also, the electronic and ionic resistances can vary with temperature, although in this particular study homogeneous temperature was assumed within the anode. The governing equations explained below were discretized using the finite volume method (FVM) and solved using an in-house Fortran program. A steady-state model of the anode was already reported in our previous reports $[12,14]$ and the model was extended to the unsteady-state analysis in this study to reproduce impedance response.

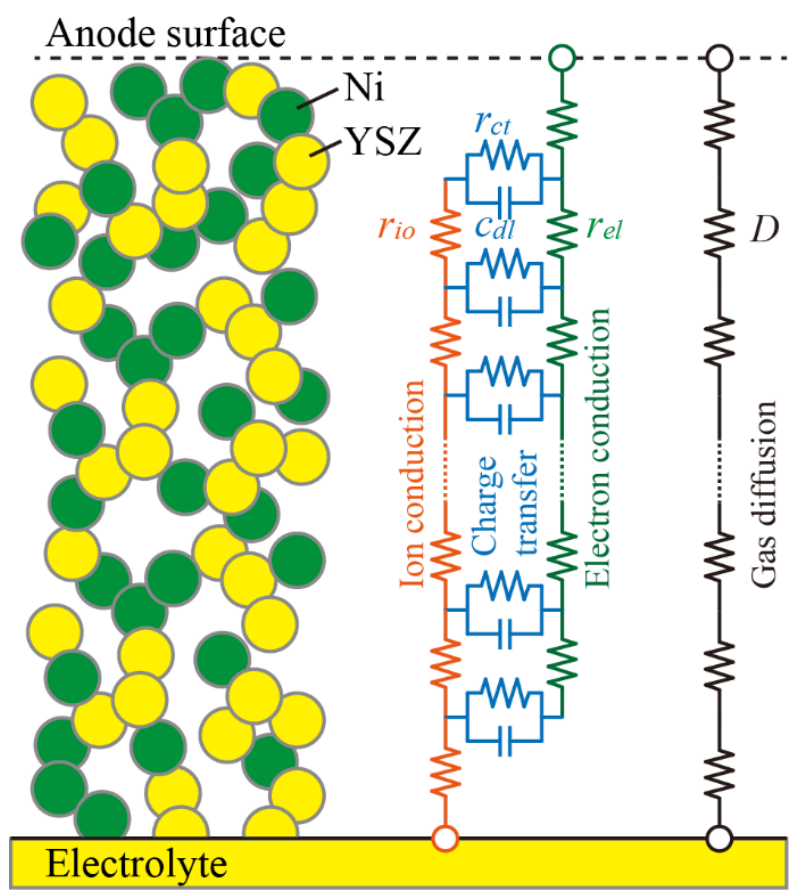

Fig. 1 Schematic picture of the calculation model. 


\subsection{Governing equations}

The conservation of electrons and ions is described as follows considering the charge and discharge current from the capacitive element in the anode.

$$
\begin{aligned}
& c_{d l} \frac{\partial}{\partial t}\left(\phi_{e l}-\phi_{i o}\right)-\nabla \cdot\left(\sigma_{e l}^{\mathrm{eff}} \nabla \phi_{e l}\right)=-i_{c t} \\
& -c_{d l} \frac{\partial}{\partial t}\left(\phi_{e l}-\phi_{i o}\right)-\nabla \cdot\left(\sigma_{i o}^{\mathrm{eff}} \nabla \phi_{i o}\right)=i_{c t}
\end{aligned}
$$

where $\phi_{e l}$ and $\phi_{i o}$ are the electric potentials of the Ni and YSZ phases, respectively; $\sigma_{e l}^{\text {eff }}$ and $\sigma_{i o}^{\text {eff }}$ are the effective electronic and ionic conductivities, respectively; $c_{d l}$ is the electrostatic capacitance; and $i_{c t}$ is the volumetric density of the charge-transfer current associated with the electrochemical reaction in the anode.

The unsteady-state conservation equation of the gas species is described as follows.

$$
\frac{V_{\text {Pore }}}{R T} \frac{\partial P_{i}}{\partial t}+\nabla \cdot N_{i}=\dot{s}_{i}
$$

where $V_{\text {Pore }}$ is the porosity of the anode; $P_{i}$ is the partial pressure; $N_{i}$ is the molar flux; and $\dot{s}_{i}$ is the sink/source term associated with the electrochemical reaction in the anode as follows.

$$
\dot{S}_{\mathrm{H}_{2}}=-\frac{i_{c t}}{2 F}, \quad \dot{s}_{\mathrm{H}_{2} \mathrm{O}}=\frac{i_{c t}}{2 F}
$$

The dusty-gas model was used to obtain the molar flux in the porous anode.

$$
\frac{N_{i}}{D_{i, K}^{\text {eff }}}+\sum_{j=1, j \neq i}^{n} \frac{X_{j} N_{i}-X_{i} N_{j}}{D_{i j}^{\text {eff }}}=-\frac{P_{t}}{R T} \nabla X_{i}-\frac{X_{i}}{R T}\left(1+\frac{K P_{t}}{\mu D_{i, K}^{\text {eff }}}\right) \nabla P_{t}
$$

where $X_{i}$ is the molar fraction; $D_{i, K}^{\text {eff }}$ and $D_{i j}^{\text {eff }}$ are the effective Knudsen and molecular diffusivities, respectively; $P_{t}$ is the total pressure; $\mu$ is the mixture viscosity; and $K$ is the permeability.

The effective conductivities and gas diffusivities were evaluated by modifying the bulk transport coefficients by using the volume fraction and the tortuosity factor of the corresponding phases. 


$$
\Gamma_{i}^{\mathrm{eff}}=\frac{V_{i}}{\tau_{i}} \Gamma_{i}, \quad\left(\Gamma \equiv \sigma_{e l}, \sigma_{i o}, D_{i j}, D_{i, K}\right)
$$

Empirical formulas were used for the bulk transport coefficients as follows $[15,16]$

$$
\begin{aligned}
& \sigma_{e l}=3.27 \times 10^{4}-1065.3 T \\
& D_{i j}=\frac{0.01013 T^{1.75}\left(\frac{1}{10^{3} M_{i}}+\frac{1}{10^{3} M_{j}}\right)^{0.5}}{P_{t}\left[\left(\Sigma v_{i}\right)^{1 / 3}+\left(\Sigma v_{j}\right)^{1 / 3}\right]^{2}} \\
& D_{i, K}=\frac{d_{\text {Pore }}}{2} \frac{2}{3} \sqrt{\frac{8 R T}{\pi M_{i}}}
\end{aligned}
$$

where $M_{i}$ is the molecular mass; $\sum v_{i}$ is the diffusion volume; and $d_{\text {Pore }}$ is the mean pore diameter. The ionic conductivity, $\sigma_{i o}$, was used as a calibration parameter in the model. Permeability of the porous anode was evaluated as follows [12].

$$
K=\frac{V_{\text {Pore }}}{6 \tau_{\text {Pore }}(S / V)_{\text {Pore }}^{2}}
$$

where $(S / V)_{\text {Pore }}$ is the surface-to-volume ratio of the pore phase. The electrostatic capacitance of the anode was assumed to linearly depend on the interfacial area between the Ni and YSZ phases [17].

$$
c_{d l}=c_{\mathrm{Ni}-\mathrm{YSZ}} S_{\mathrm{Ni}-\mathrm{YSZ}}
$$

where $S_{\mathrm{Ni}-\mathrm{YSZ}}$ is the volumetric density of the Ni-YSZ interfacial area, and $c_{\mathrm{Ni}-\mathrm{YSZ}}$ is the electrostatic capacitance per unit Ni-YSZ interfacial area.

The electrochemical oxidation of hydrogen was assumed to take place at the TPBs, and described by the non-linear Butler-Volmer-type equation as follows [18].

$$
i_{c t}=i_{0, \mathrm{TPB}} l_{\mathrm{TPB}}\left[\exp \left(\frac{2 F}{R T} \eta_{\text {act }}\right)-\exp \left(-\frac{F}{R T} \eta_{\text {act }}\right)\right]
$$

where the exchange current density was considered as a function of the TPB density $l_{\text {TPB }}$. The exchange current per unit TPB length, $i_{0, \mathrm{TPB}}$, was used as a fitting parameter in the model. The driving force of the 
electrochemical reaction is the activation overpotential and was evaluated as follows.

$$
\eta_{\text {act }}=\phi_{e l}-\phi_{i o}-\eta_{\text {con }}
$$

where $\eta_{\text {con }}$ is the concentration overpotential as follows.

$$
\eta_{\text {con }}=\frac{R T}{2 F} \ln \left(\frac{P_{\mathrm{H}_{2}}^{\text {bulk }}}{P_{\mathrm{H}_{2}}} \frac{P_{\mathrm{H}_{2} \mathrm{O}}}{P_{\mathrm{H}_{2} \mathrm{O}}^{\text {bulk }}}\right)
$$

\section{$2.3 \quad$ Boundary conditions}

Boundary conditions are summarized in Table $1\left(L_{a}\right.$ is the anode thickness). Constant gas partial pressures were applied on the anode surface. No flux condition was applied to the gas partial pressures and the electric potential in the Ni phase at the anode-electrolyte interface, and to the electric potential in the YSZ phase at the anode surface. For the impedance simulation, an electric potential with sinusoidal perturbation was imposed to the electric potential in the Ni phase at the anode surface, with an amplitude of $\eta_{p}$ and an angular velocity of $\omega=2 \pi f$.

Table 1 Boundary conditions.

\begin{tabular}{lcc} 
Variables & Surface $\left(x=L_{a}\right)$ & Interface $(x=0)$ \\
\hline $\mathrm{H}_{2}$ partial pressure & $P_{\mathrm{H}_{2}}\left(L_{a}\right)=P_{\mathrm{H}_{2}}^{\text {bulk }}$ & $\frac{d P_{\mathrm{H}_{2}}}{d x}(0)=0$ \\
$\mathrm{H}_{2} \mathrm{O}$ partial pressure & $P_{\mathrm{H}_{2} \mathrm{O}}\left(L_{a}\right)=P_{\mathrm{H}_{2} \mathrm{O}}^{\text {bulk }}$ & $\frac{d P_{\mathrm{H}_{2} \mathrm{O}}}{d x}(0)=0$ \\
Electric potential in Ni & $\phi_{e l}\left(L_{a}\right)=\eta_{s}+\eta_{p} \sin \omega t$ & $\frac{d \phi_{e l}}{d x}(0)=0$ \\
Electric potential in YSZ & $\frac{d \phi_{i o}}{d x}\left(L_{a}\right)=0$ & $\phi_{i o}(0)=0$ \\
\hline
\end{tabular}




\section{$2.4 \quad$ Impedance calculation}

First, the steady state solution was obtained without the perturbation, i.e., $\phi_{\mathrm{el}}\left(L_{a}\right)=\eta_{s}$, then the unsteady-state simulation was conducted with the perturbation for several periods until the current response curve became identical between the neighboring periods. The amplitude of the perturbation $\eta_{p}$ was set as $5 \mathrm{mV}$. The time step used in this study was $1 / 256$ of each period. The complex impedance of the anode was obtained as follows.

$$
Z(\omega)=\frac{\eta_{p}}{\operatorname{Re}\{I(\omega)\}+j \operatorname{Im}\{I(\omega)\}}
$$

where $\operatorname{Re}\{I(\omega)\}$ and $\operatorname{Im}\{I(\omega)\}$ were obtained by integrating the time-dependent current response of the anode, $I(t)$, with sine and cosine functions.

$$
\begin{aligned}
& \operatorname{Re}\{I(\omega)\}=\frac{2}{\theta} \int_{0}^{\theta} I(t) \sin (\omega t) d t \\
& \operatorname{Im}\{I(\omega)\}=\frac{2}{\theta} \int_{0}^{\theta} I(t) \cos (\omega t) d t
\end{aligned}
$$

where $\theta=1 / f$ is the period of the sinusoidal perturbation.

\section{$3 \quad$ Experiment}

To calibrate the numerical model developed in this study, impedance measurement of an anode was conducted on the conventional electrolyte-supported cells consisting of a Ni-YSZ anode, a YSZ electrolyte, a GDC interlayer and an LSCF cathode.

\subsection{Sample preparation}

A mixture of NiO powder (FUJIFILM Wako Pure Chemical Corp., Japan) and YSZ powder (TZ-8Y, 
Tosoh Corp., Japan) was prepared and mixed with a terpineol-based binder (VEH, Nexceris, LLC, U.S.A., powder: binder $=75: 25 \mathrm{wt} . \%)$ to obtain anode slurry. The slurry was screen-printed on a YSZ disk electrolyte (thickness: $500 \mu \mathrm{m}$, diameter: $24 \mathrm{~mm}$, Tosoh Corp., Japan), and sintered at $1,450^{\circ} \mathrm{C}$ for $5 \mathrm{~h}$. The GDC powder (Shin-Etsu Astech Co., Ltd., Japan) was mixed with the same binder and screen printed on the other side of the electrolyte and sintered at $1,350^{\circ} \mathrm{C}$ to form an interlayer to prevent the reaction between the $\mathrm{YSZ}$ electrolyte and the LSCF cathode. Then, on the GDC interlayer, LSCF slurry (LSCF-I, Fuelcellmaterials, U.S.A.) was screen-printed and sintered at $1,150^{\circ} \mathrm{C}$ for $5 \mathrm{~h}$.

In this study, two samples were prepared. For the one sample (Anode I), the NiO-YSZ mixed powder was rigorously milled with a planetary mill with zirconia balls for $1 \mathrm{~h}$ to reduce the particle sizes. This was used for calibration of the numerical model. For the other sample (Anode II), the mixed powder was gently potmilled in ethanol so that the powders were well-dispersed, but their particle sizes were as-provided. The YSZ electrolyte, the GDC interlayer and the LSCF cathode of the two samples were identical. The thickness of the anode was 10 and $30 \mu \mathrm{m}$ for Anode I and Anode II, respectively.

\subsection{Impedance measurement}

For the EIS measurement, the prepared cells were held between two aluminum tubes with platinum mesh for current collection. The cell temperature was controlled by an electronic furnace from 600 to $800^{\circ} \mathrm{C}$. $3 \%$ humidified hydrogen was supplied to the anode side, whereas synthetic dry air to the cathode side. More details of the test system can be found elsewhere [12]. 
Impedance characteristics of the cells were obtained by using Celltest System 1470E with a frequency response analyzer 1455A (Solartron Analytical, U.K.). The amplitude of the perturbation current was $1 \mathrm{~mA}$ and its frequency range was from $10^{6}$ to $10^{-1} \mathrm{~Hz}$. Impedance characteristics were obtained not only at the open circuit conditions but also at several current bias conditions.

\subsection{Equivalent circuit fitting}

When the overpotential applied to the anode is small enough, i.e., near the open circuit conditions, the TLM can be reasonably used as an equivalent circuit for the anode because gas concentration, local activation resistance, and conductivities are assumed to be uniform within the entire anode. The TLM impedance is obtained as an analytical solution of eqs. (1), (2) and (12) as follows [8].

$$
\begin{gathered}
Z_{T L M}=\frac{L_{a}}{\Gamma \sinh \Gamma}\left(\frac{1}{\sigma_{e l}^{\text {eff }}}+\frac{1}{\sigma_{i o}^{\text {eff }}}\right)\left[\cosh \Gamma+\frac{\sigma_{e l}^{\text {eff }} \sigma_{i o}^{\text {eff }}}{\left(\sigma_{e l}^{\text {eff }}+\sigma_{i o}^{\text {eff }}\right)^{2}}(2+\Gamma \sinh \Gamma+2 \cosh \Gamma)\right] \\
\Gamma^{2} \equiv L_{a}^{2}\left[\frac{3 F i_{0, \mathrm{TPB}} l_{\mathrm{TPB}}}{R T}+j \omega c_{d l}\right]\left(\frac{1}{\sigma_{e l}^{\text {eff }}}+\frac{1}{\sigma_{i o}^{\text {eff }}}\right)
\end{gathered}
$$

Given that the electronic conductivity is a few orders of magnitude larger than the ionic conductivity in the typical SOFC anodes, the TLM impedance can be simplified as follows.

$$
Z_{\mathrm{TLM}}=\sqrt{\frac{r_{i o} r_{c t}}{1+j \omega r_{c t} c_{d l}}} \operatorname{coth}\left[L_{a} \sqrt{\frac{r_{i o}}{r_{c t}}\left(1+j \omega r_{c t} c_{d l}\right)}\right]
$$

where $r_{c t}=R T / 3 F i_{0, \mathrm{TPB}} l_{\mathrm{TPB}}$ and $r_{i o}=1 / \sigma_{i o}^{\text {eff }}$. It should be noted that the impedance in eq. (20) equals the finite-length Gerischer element. In this study, the impedance spectra obtained in the experiment were fitted to a serial circuit of an inductance, an ohmic resistance and the TLM in eq. (20) to obtain the ionic resistance $r_{i o}$, the charge-transfer (activation) resistance $r_{c t}$, and the electrostatic capacitance $c_{d l}$ of the anodes. They were 
then correlated with the parameters used in the numerical model as follows.

$$
\begin{gathered}
\sigma_{i o}=\frac{1}{r_{i o}} \frac{\tau_{i o}}{V_{i o}} \\
i_{0, \mathrm{TPB}}=\frac{R T}{3 F l_{\mathrm{TPB}}} \frac{1}{r_{c t}} \\
c_{\mathrm{Ni}-\mathrm{YSZ}}=\frac{c_{d l}}{S_{\mathrm{Ni}-\mathrm{YSZ}}}
\end{gathered}
$$

\subsection{Microstructural analysis}

After the impedance measurements, the cells were provided for microstructural analysis using the focused ion beam scanning electron microscope (FIB-SEM). The cells were first impregnated with epoxy resin (EpoFix, Struers, Denmark) under vacuum to allow easier identification of the pore phase in the subsequent FIB-SEM imaging. The three-dimensional (3D) microstructure of the anodes was imaged using an NVision 40 (Carl Zeiss, Germany) FIB-SEM system. The in-lens secondary electron detector was used to distinguish the two solid phases, i.e., Ni and YSZ, in the SEM images. After the imaging, the slice images were aligned and segmented into the three phases and reconstructed to 3D microstructure for quantification. In this study, volume fractions, tortuosity factors, particle/pore sizes, Ni-YSZ interfacial area density and TPB density were quantified. Details of the image processing procedures and the quantification methodologies can be found elsewhere [19, 20]. 


\section{Results and Discussion}

4.1 Microstructural parameters

The 3D microstructure of the tested anodes is reconstructed and quantified. Fig. 2 shows the segmented cross-sectional images and the 3D reconstructed structures of Anode I and Anode II. The reconstructed volume size is $9.2 \times 9.9 \times 6.4 \mu \mathrm{m}^{3}$ for Anode I and $23.2 \times 18.3 \times 12.8 \mu \mathrm{m}^{3}$ for Anode II. Microstructural parameters quantified from Anode I and Anode II are shown in Table 2. The solid volume ratio of Ni to YSZ is close to unity, which partially assures the representativeness of the reconstructed volumes. Since the particles sizes in Anode II is larger than those in Anode I, more pores are formed in the porous structure and the Ni-YSZ interfacial area and the TPB density are significantly decreased in Anode II.
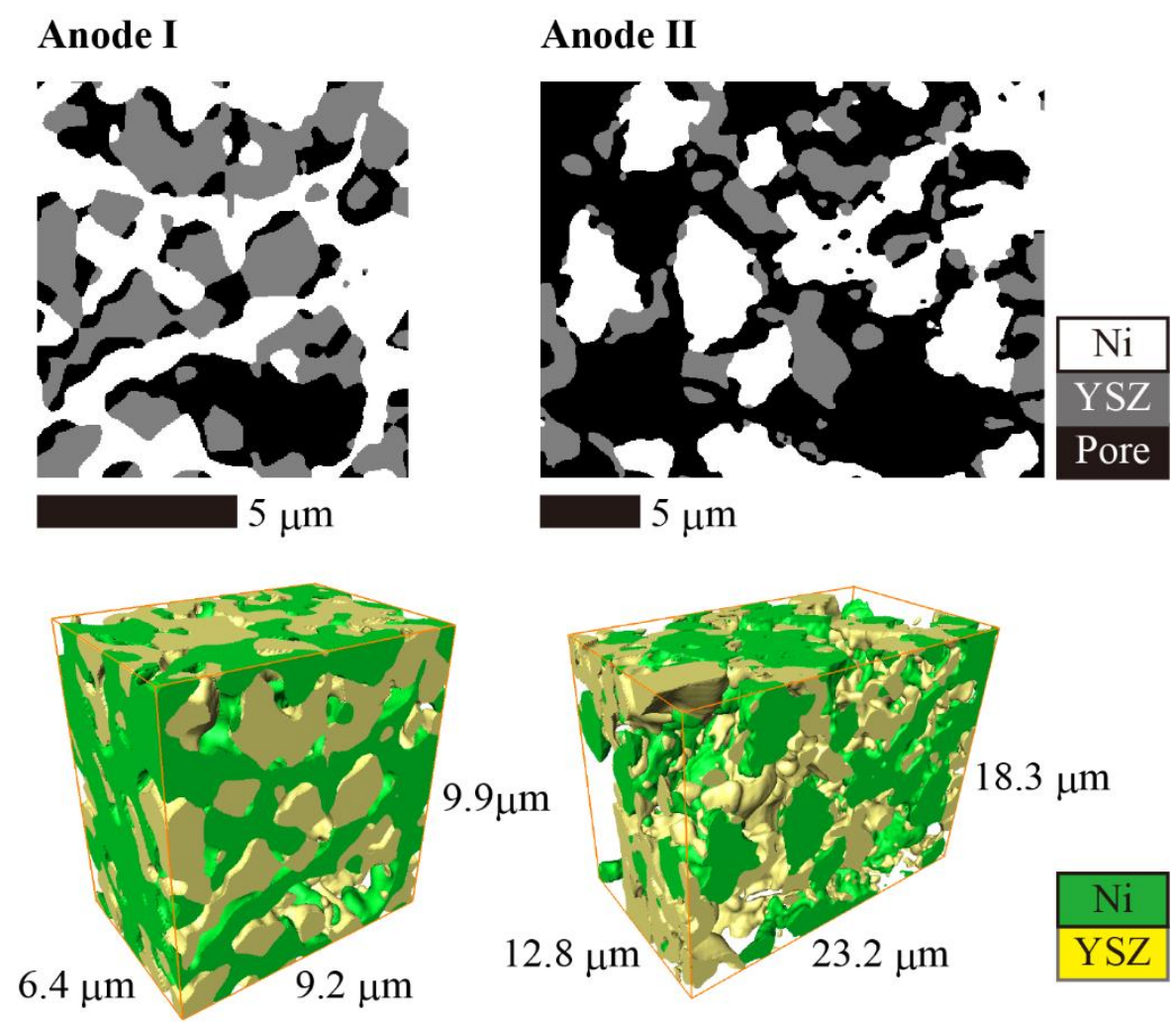

Fig. 2 Cross-sectional images and 3D reconstructed structures of Anode I and Anode II. 
Table 2 Microstructural parameters quantified from Anode I and II.

\begin{tabular}{lcccccccc}
\hline & & & \multicolumn{3}{c}{ Anode I } & \multicolumn{3}{c}{ Anode II } \\
& & Unit & Ni & YSZ & Pore & Ni & YSZ & Pore \\
\hline Volume fraction & $V_{i}$ & {$[-]$} & 0.389 & 0.368 & 0.243 & 0.220 & 0.237 & 0.543 \\
Particle/pore size & $d_{i}$ & {$[\mu \mathrm{m}]$} & 1.47 & 1.21 & 0.961 & 2.82 & 2.92 & 5.22 \\
Tortuosity factor & $\tau_{i}$ & {$[-]$} & 7.15 & 3.64 & 7.93 & 7.37 & 9.35 & 1.73 \\
Ni-YSZ interfacial area & $S_{\mathrm{Ni}-\mathrm{YSZ}}$ & {$\left[\mu \mathrm{m}^{2} / \mu \mathrm{m}^{3}\right]$} & & 0.745 & & & 0.0870 & \\
TPB density & $l_{\mathrm{TPB}}$ & {$\left[\mu \mathrm{m} / \mu \mathrm{m}^{3}\right]$} & & 2.45 & & & 0.383 & \\
\hline
\end{tabular}

\subsection{Model calibration}

Impedance spectra of Anode I is obtained at the open circuit conditions at the temperature from 600 to $800^{\circ} \mathrm{C}$, and fitted to the equivalent circuit model including the TLM. The measured impedance spectra and the fitting curves are shown in Fig. 3. Even though the impedance arcs on the Nyquist plot are not the perfect semicircles, they can be accurately reproduced using the conventional equivalent circuit model. Also, the position of the peak in Fig. 3(b), which is related with the characteristic time scale of the electrode kinetics, is also accurately reproduced by properly adjusting the electrostatic capacitance of the anode. This re-ensures the validity of the original TLM near the open circuit conditions.

(a)

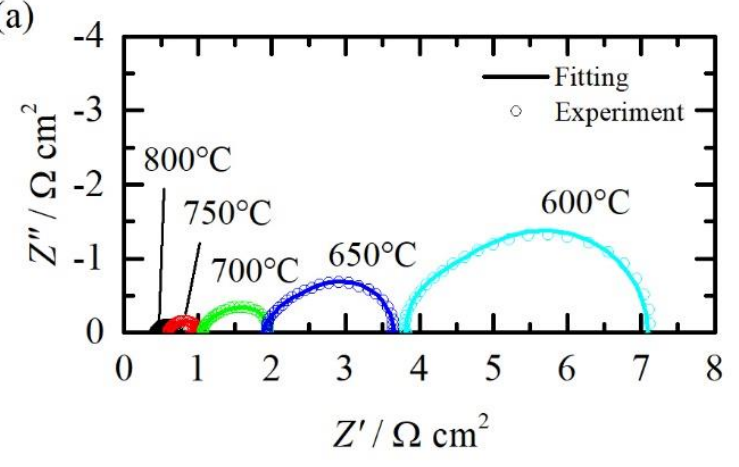

(b)

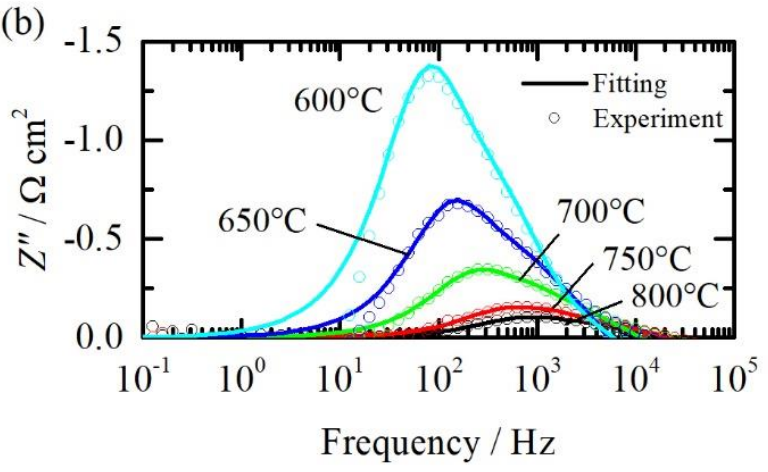

Fig. 3 Experimental and fitted impedance spectra of Anode I at the open circuit conditions. (a) Nyquist plot 
and (b) Bode plot of the imaginary component.

The obtained parameters from the equivalent circuit fitting are summarized in Table 3, and shown on the Arrhenius plot in Fig. 4 for the ionic conductivity $\sigma_{i o}$, the exchange current density $i_{0, T P B}$ and the electrostatic capacitance $c_{\mathrm{Ni}-\mathrm{YSZ}}$. It is confirmed that the temperature dependency of the three parameters follows the Arrhenius-type formula and can be expressed as follows.

$$
\begin{aligned}
& \sigma_{i o}=\frac{1}{r_{i o}} \frac{\tau_{i o}}{V_{i o}}=9.37 \times 10^{3} \exp \left(-\frac{7.77 \times 10^{4}}{R T}\right) \\
& i_{0, \mathrm{TPB}}=\frac{R T}{3 F l_{\mathrm{TPB}}} \frac{1}{r_{c t}}=96.7 \exp \left(-\frac{1.23 \times 10^{5}}{R T}\right) \\
& c_{\mathrm{Ni}-\mathrm{YSZ}}=\frac{c}{S_{\mathrm{Ni}-\mathrm{YSZ}}}=155 \exp \left(-\frac{3.61 \times 10^{4}}{R T}\right)
\end{aligned}
$$

The activation energy of the ionic conductivity is found to be $77.7 \mathrm{~kJ} \mathrm{~mol}^{-1}$, which is close to the reported values of $84 \sim 87 \mathrm{~kJ} \mathrm{~mol}^{-1}$ in ref. [21-23]. The activation energy of the exchange current density is found to be $123 \mathrm{~kJ}$ $\mathrm{mol}^{-1}$, which is between the reported values of $152 \mathrm{~kJ} \mathrm{~mol}^{-1}$ by de Boer [24] and $85 \mathrm{~kJ} \mathrm{~mol}^{-1}$ by Bieberle et al. [25]. The activation energy of the electrostatic capacitance is found to be $36.1 \mathrm{~kJ} \mathrm{~mol}^{-1}$, which is within the reported values of $13 \sim 51 \mathrm{~kJ} \mathrm{~mol}^{-1}$ in ref. $[17,26,27]$. These can assure reliability of the measurements and the equivalent circuit fitting conducted in this study.

The anode numerical analysis is then conducted at the open circuit conditions to reproduce the impedance characteristics using the quantified microstructural parameters in Table 2 and the eqs. (24-26). The results (not shown) are confirmed to be identical with the experimental results and the fitting curves shown in Fig. 3. This is obvious because at the open circuit conditions, the developed numerical model is essentially 
equivalent to the TLM in eq. (18).

Table 3 Fitted parameters for Anode I.

\begin{tabular}{|c|c|c|c|c|c|}
\hline $\begin{array}{l}T \\
{\left[{ }^{\circ} \mathrm{C}\right]}\end{array}$ & $\begin{array}{l}L \\
{[\mathrm{nH}]}\end{array}$ & $\begin{array}{l}R_{h} \\
{\left[\Omega \mathrm{cm}^{2}\right]}\end{array}$ & $\begin{array}{l}r_{i o} \\
{[\Omega \mathrm{cm}]}\end{array}$ & $\begin{array}{l}r_{c t} \\
{\left[\Omega \mathrm{cm}^{3}\right]}\end{array}$ & $\begin{array}{l}c_{d l} \\
{\left[\mathrm{~F} \mathrm{~cm}^{-3}\right]}\end{array}$ \\
\hline 800 & $2.34 \times 10^{2}$ & 0.390 & $6.32 \times 10^{2}$ & $1.43 \times 10^{-4}$ & 1.93 \\
\hline 750 & $3.05 \times 10^{2}$ & 0.550 & $9.84 \times 10^{2}$ & $2.03 \times 10^{-4}$ & 1.86 \\
\hline 700 & $8.22 \times 10^{2}$ & 0.942 & $1.61 \times 10^{3}$ & $5.63 \times 10^{-4}$ & 1.27 \\
\hline 650 & $5.89 \times 10^{3}$ & 1.68 & $2.55 \times 10^{3}$ & $1.24 \times 10^{-3}$ & 0.943 \\
\hline 600 & $3.68 \times 10^{3}$ & 3.18 & $4.75 \times 10^{3}$ & $2.49 \times 10^{-3}$ & 0.841 \\
\hline
\end{tabular}

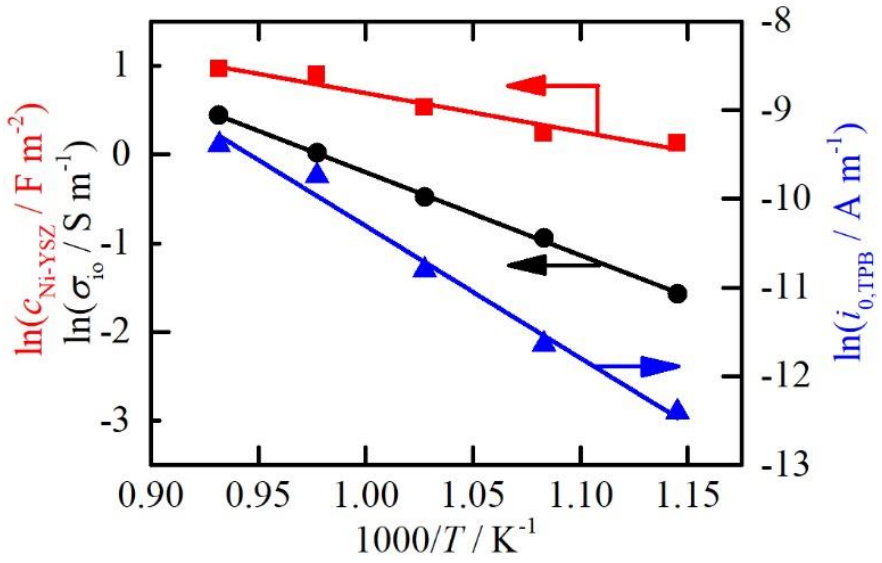

Fig. 4 Arrhenius plots of the ionic conductivity, the exchange current density and the electrostatic capacitance

of Anode I in 3\% humidified hydrogen.

\subsection{Impedance at the polarized conditions}

By using the calibrated numerical model, impedance spectra at the polarized conditions are obtained and

compared with the experiment as shown in Fig. 5. The impedance spectra are measured with Anode I at several current-biased conditions up to $60 \mathrm{~mA} \mathrm{~cm}{ }^{-2}$, which corresponds to the anode overpotential up to ca. $500 \mathrm{mV}$ at $700^{\circ} \mathrm{C}$. It is found that the developed model can accurately reproduce the impedance spectra not only at the 
open circuit conditions but also at the polarized conditions. Note that the bulk ohmic resistance is subtracted from the impedance spectra to exclude the contributions of the electrolyte resistance and the contact resistance, which are not considered in the numerical model. The discrepancy between the experiment and the simulation found in the high frequency region is due to the parasitic inductance in the experiment.

Fig. 5(b) indicates that the position of the peak in the imaginary component of the impedance is shifted towards the high frequency as the current bias is increased; this trend is also accurately reproduced with the developed model without re-tuning the parameters such as the electrostatic capacitance. This result cannot be obtained with the conventional TLM because the ionic resistance, the activation resistance and the electrostatic capacitance are not allowed to vary with the current/voltage bias. Fig. 6 shows the distribution of the local activation resistance $r_{c t}$ in Anode I at $700^{\circ} \mathrm{C}$ calculated as follows.

$$
r_{c t}=\frac{\partial \eta_{\mathrm{act}}}{\partial i_{\mathrm{ct}}}=\frac{R T}{i_{0, \mathrm{TPB}} l_{\mathrm{TPB}} F}\left[2 \exp \left(\frac{2 F}{R T} \eta_{\mathrm{act}}\right)+\exp \left(-\frac{F}{R T} \eta_{\mathrm{act}}\right)\right]^{-1}
$$

This result clearly shows the non-uniform distribution of the activation resistance at the polarized conditions.

The activation resistance in the vicinity of the anode-electrolyte interface is decreased as the polarization increased.

The position of the peak in the Bode plot in Fig. 5(b) is known to correspond to the characteristic time scale and be inversely proportional to the product of the charge-transfer resistance and the electrostatic capacitance. Therefore Fig. 5(b) indicates that the charge-transfer resistance and/or the electrostatic capacitance decreases as the anode is polarized. According to eq. (27), it is easily confirmed that the charge-transfer resistance decreases as the anode is polarized. Since this inherent characteristic of the charge-transfer resistance 
can fully reproduce the impedance characteristics in the Bode plot in Fig. 5(b), the electrostatic capacitance of

$\mathrm{Ni}-\mathrm{YSZ}$ anodes is found to be irrespective of the polarization state of the anodes.

(a)

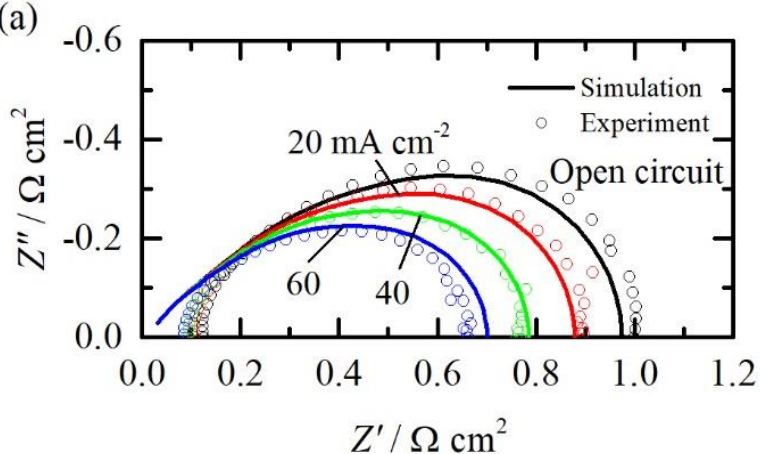

(b)

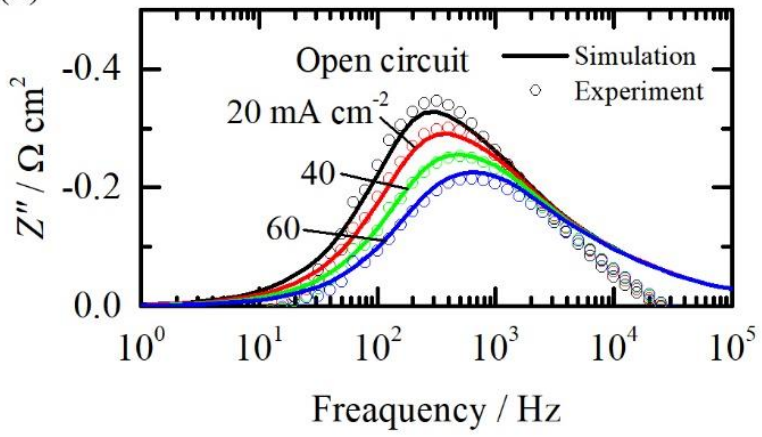

Fig. 5 Comparison of the simulated impedance spectra and the experimental results with Anode I at the open circuit condition and with the current density up to $60 \mathrm{~mA} \mathrm{~cm}^{-2}$ at $700^{\circ} \mathrm{C}$. (a) Nyquist plot and (b) Bode plot of the imaginary component.

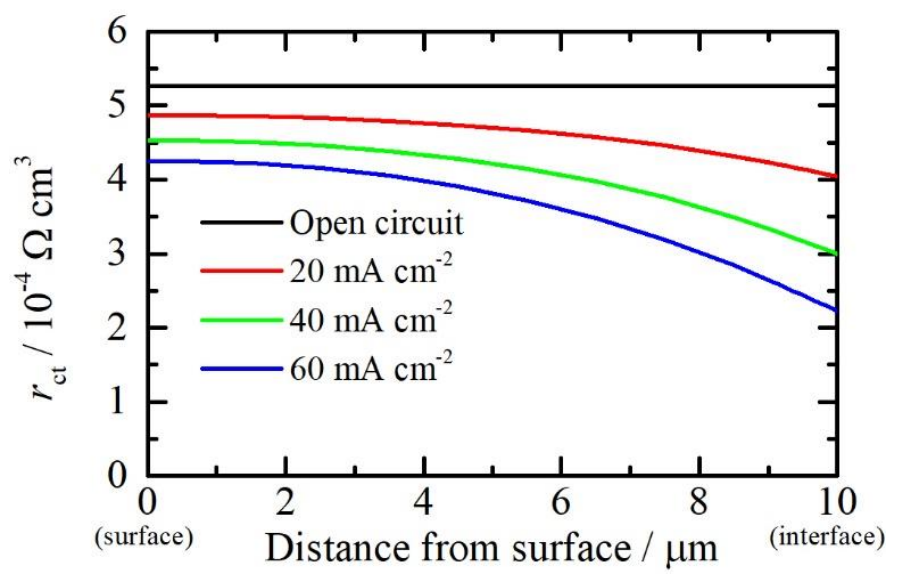

Fig. 6 Distribution of the local activation resistance in Anode I at $700^{\circ} \mathrm{C}$.

\subsection{Impedance of an anode with different microstructure}

To further validate the generality of the developed numerical model, the model is used to simulate the impedance response obtained from another anode with a different microstructure (Anode II). The impedance 
spectra are obtained from Anode II at the temperatures from $600^{\circ} \mathrm{C}$ to $800^{\circ} \mathrm{C}$ at the open circuit conditions. The anode microstructural parameters are also quantified from the reconstructed 3D microstructure of Anode II (Table 2), and implemented in the numerical model. It should be emphasized again that the microstructural parameters are not used as fitting parameters in this analysis. Fig. 7 shows the comparison between the simulation results and the experimental results obtained from Anode II. It is found from Fig. 7(a) that the impedance semi-circles are well reproduced on the Cole-Cole plot, even though the model does not have any arbitrary fitting parameters. However, the peaks in Fig. 7(b) obtained in the simulation are slightly shifted towards the right from those in the experiment. Since the peak frequency is proportional to the product of the charge-transfer resistance $r_{c t}$ and the electrostatic capacitance $c_{d l}$, and the trend of the charge-transfer resistance is well-reproduced as shown in Fig. 7(a), the source of the error in Fig. 7(b) is possibly from the evaluation of the electrostatic capacitance. In this study, the electrostatic capacitance in the anodes is assumed as a function of the Ni-YSZ interfacial area as in eq. (11). From Table 2, the Ni-YSZ interfacial area in Anode II is significantly less than that in Anode I, however, the size of the individual interfacial area in Anode II is much larger because of the larger particle sizes of the Ni and YSZ phases. This may indicate that not entire region of the Ni-YSZ interfacial area acts as the electrostatic capacitance when the size of the area is large. The relationships between the anode microstructure and the electrostatic capacitance is still unclear and further investigation is required. Nonetheless, the degree of agreement achieved in this study is satisfactory considering the fact that the model does not use any arbitrary fitting parameters that are freely adjustable for each anode and operation condition. 

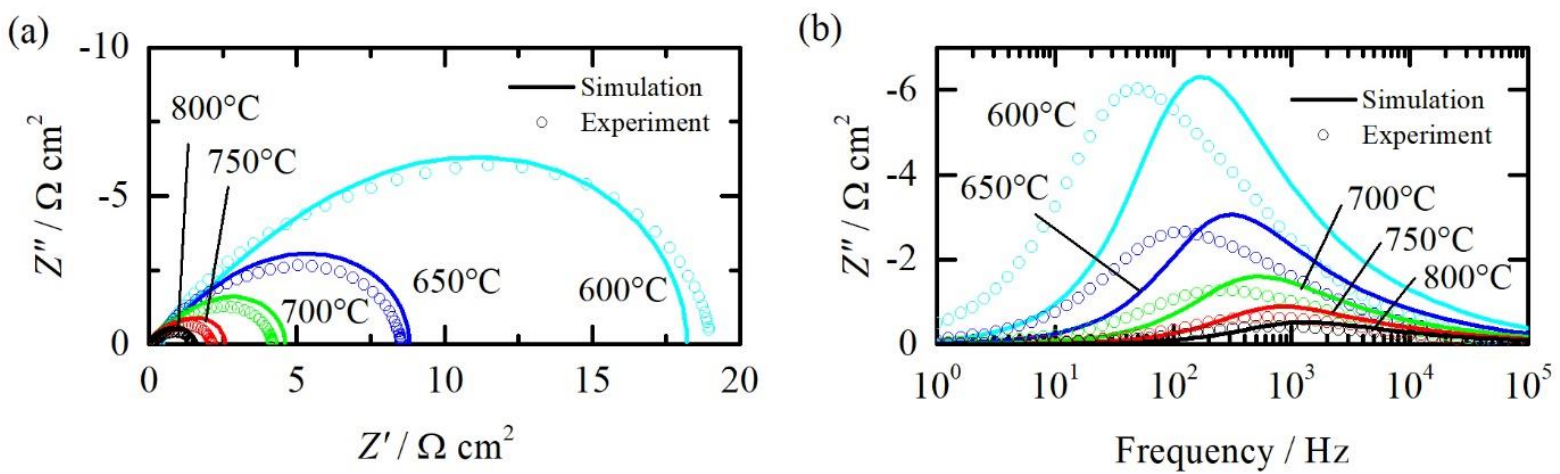

Fig. 7 Comparison of the simulated impedance spectra and experimental results with Anode II at the open circuit conditions for different temperatures. (a) Nyquist plot and (b) Bode plot of the imaginary component.

\subsection{Impedance analysis with microstructure estimation}

As discussed above, the developed numerical model can reasonably reproduce the anode impedance spectra not only at the open circuit conditions but also at the polarized conditions by applying the actual microstructural parameters of the anodes. However, in the real situations, microstructural parameters of the anodes are not always available because the 3D microstructural analysis using the FIB-SEM is extensively timeconsuming and costly. In addition, because of the destructive nature of the FIB-SEM analysis, the anode microstructure will not be available during long-term operation tests. Therefore, for more practical application of the developed model, the model can be calibrated with the impedance spectra to assume microstructural parameters of the anodes.

In this study, an additional anode was fabricated using the same NiO-YSZ paste as used for Anode I, although the sintering temperature was decreased to $1,350^{\circ} \mathrm{C}$ to have different, and hence unknown, microstructure (Anode III). The numerical model was calibrated with the impedance spectra obtained at the 
open circuit condition at $700^{\circ} \mathrm{C}$ with Anode III. As a result, the set of microstructures related with the ionic conduction is determined as $V_{\mathrm{YSZ}} / \tau_{\mathrm{YSZ}}=0.132$, the TPB density as $L_{\mathrm{TPB}}=0.834 \mu \mathrm{m}^{-3}$ and the Ni-YSZ interfacial area density as $S_{\mathrm{Ni}-\mathrm{YSZ}}=0.781 \mu \mathrm{m}^{2} \mu \mathrm{m}^{-3}$. After that, the numerical analysis is conducted with current bias up to $60 \mathrm{~mA} \mathrm{~cm}{ }^{-2}$ at 650,700 and $750^{\circ} \mathrm{C}$, and the results are shown in Fig. 8. It is found that the impedance characteristics obtained from the experiment are reasonably reproduced by the developed model with the maximum error no more than $15 \%$. It should be emphasized that the frequency dependence of the impedance is almost perfectly reproduced in terms of the characteristic time scale (corresponding to the peak frequency in the Bode plot).

These results clearly show the versatility of the developed numerical model to detect possible degradation phenomena occurring within SOFC anodes. Once the model is calibrated with the experimental data of impedance spectra at the open circuit condition, preferably at the beginning of a long-term durability test, the model can be used to reproduce the impedance spectra during the durability test with current/voltage bias. When degradation occurs and hence the shape of the impedance spectra is altered, the developed model can be used as similar to the conventional equivalent circuit models to identify the origin of the degradation within the anode.

It should be noted that the time required to reproduce impedance spectra at one operation condition is less than 1 min using a single core of Intel Xeon E5-2667 v3 CPU in a Linux workstation, or around 2 min using a single core of Intel Core i7-7700 CPU in an office-use Windows computer. Therefore, parametric investigation varying electrochemical properties is possible and the time required for this purpose is not significantly longer 
than the conventional equivalent circuit fitting approaches.

(a)

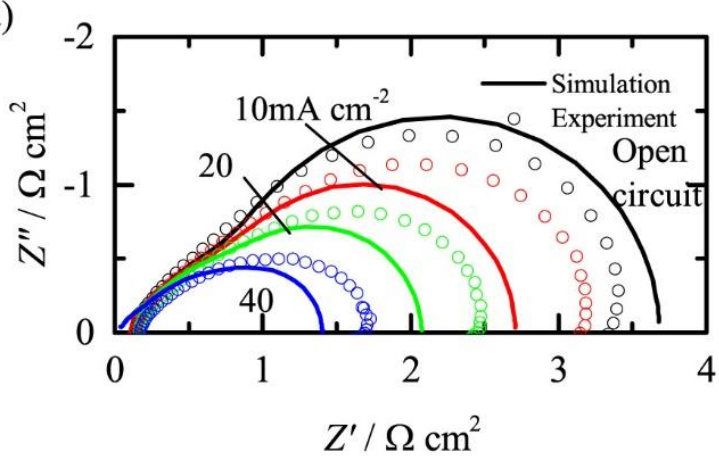

(b)

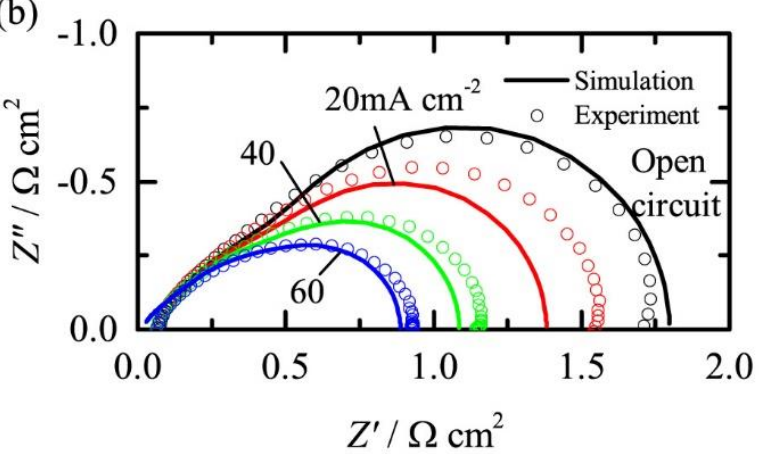

(c)

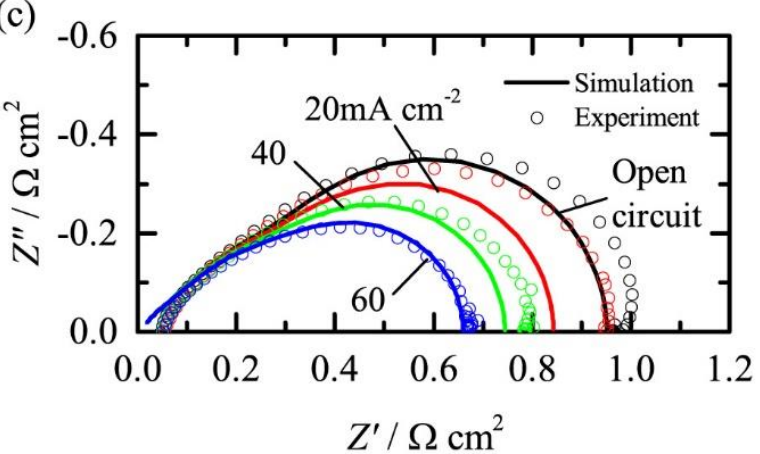

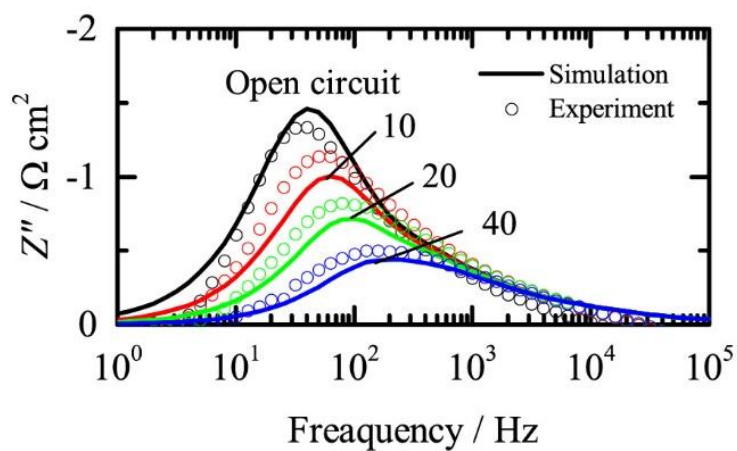

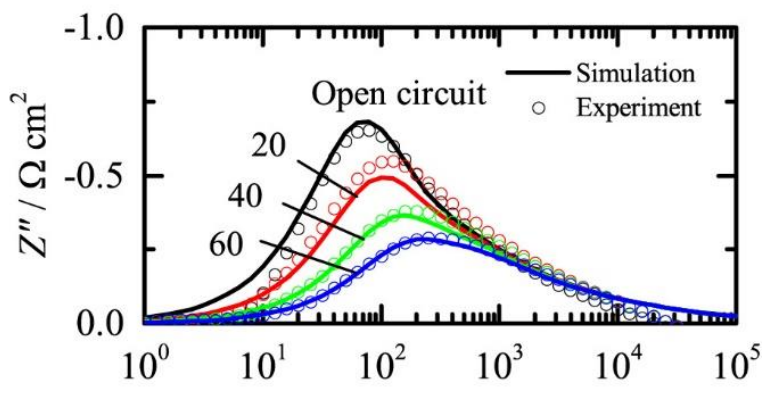

Freaquency / Hz

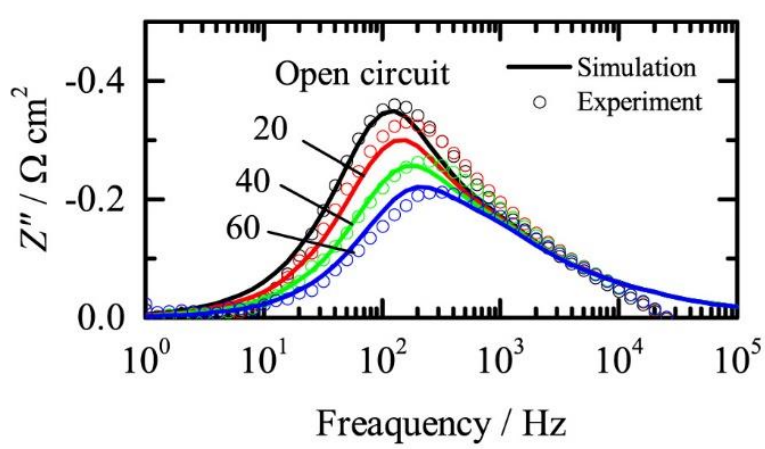

Fig. 8 Comparison of the simulated impedance spectra and experimental results with Anode III at (a) $650^{\circ} \mathrm{C}$,

(b) $700^{\circ} \mathrm{C}$ and (c) $750^{\circ} \mathrm{C}$ with the current density up to $60 \mathrm{~mA} \mathrm{~cm}{ }^{-2}$. 


\section{Conclusions}

A 1D unsteady-state numerical model of a Ni-YSZ anode of SOFCs has been developed to reproduce impedance spectra not only at the open circuit conditions but also at the polarized conditions. The model considers the conservations of electrons, ions and gas species within the porous anode, which are coupled by the electrochemical reaction at the TPBs. A Butler-Volmer like equation is used for the electrochemical reaction model, which describes a non-linear relationship between the local activation overpotential and the chargetransfer current. The real microstructural parameters are obtained from the anodes used in this study by using FIB-SEM and implemented in the numerical model to eliminate arbitrary fitting parameters. The numerical model is calibrated with the impedance spectra measured at the open circuit conditions and used to simulate those at the polarized conditions. Good agreement has been achieved both in the Cole-Cole plot and in the Bode plot at $700^{\circ} \mathrm{C}$. Also, it is suggested that the electrostatic capacitance is irrespective of the anode polarization state.

The calibrated numerical model is also proved to be able to reproduce the impedance spectra obtained from a different anode if a proper set of microstructural parameters is applied. The impedance spectra obtained from the different anode with coarser microstructure are successfully reproduced in the temperature range between 600 to $800^{\circ} \mathrm{C}$. Moreover, even if the real anode microstructure is unknown, by estimating the microstructural parameters from the fitting at the open circuit condition at $700^{\circ} \mathrm{C}$, the developed numerical model can reasonably reproduce the impedance spectra at the different temperatures from 650 to $750^{\circ} \mathrm{C}$ and also with the current bias up to $60 \mathrm{~mA} \mathrm{~cm}^{-2}$. These results ensure versatility of the developed numerical model. 
Although the concept of the developed anode model is relatively simple, it is rigorously based on the

physicochemical processes in the anode, such as the charge conduction, the gas diffusion and the electrochemical reaction. In addition, since it takes less than a few minutes to reproduce impedance spectra at one operation condition, the model can be used as an alternative to the conventional equivalent circuit fitting approaches.

\section{Acknowledgment}

This work was supported under "Fundamental study on rapid evaluation method of SOFC durability" by the New Energy and Industrial Technology Organization (NEDO, Japan). 


\section{Nomenclature}

$c_{d l} \quad$ electrostatic capacitance $\left[\mathrm{F} \mathrm{m}^{-3}\right]$

$c_{\mathrm{Ni}-\mathrm{YSZ}}$ electrostatic capacitance for unit Ni-YSZ interfacial area $\left[\mathrm{F} \mathrm{m}^{-2}\right]$

$D_{i j} \quad$ molecular diffusivity $\left[\mathrm{m}^{2} \mathrm{~s}^{-1}\right]$

$D_{i, K} \quad$ Knudsen diffusivity $\left[\mathrm{m}^{2} \mathrm{~s}^{-1}\right]$

$d_{i} \quad$ particle/pore diameter $[\mu \mathrm{m}]$

F $\quad$ Faraday constant $\left[\mathrm{C} \mathrm{mol}^{-1}\right]$

$f \quad$ frequency $\left[\mathrm{s}^{-1}\right]$

I current density $\left[\mathrm{A} \mathrm{m}^{-2}\right]$

$i_{c t} \quad$ charge-transfer current density $\left[\mathrm{A} \mathrm{m}^{-3}\right]$

$i_{0, \mathrm{TPB}} \quad$ exchange current per unit TPB length $\left[\mathrm{A} \mathrm{m}^{-1}\right]$

$K \quad$ permeability $\left[\mathrm{m}^{2}\right]$

$L \quad$ inductance $[\mathrm{H}]$

$L_{a} \quad$ anode thickness [m]

$l_{\text {TPB }} \quad$ TPB density $\left[\mathrm{m}^{-2}\right]$

$M_{i} \quad$ molecular mass $\left[\mathrm{kg} \mathrm{mol}^{-1}\right]$

$N_{i} \quad$ molar flux $\left[\mathrm{mol} \mathrm{m}^{-2} \mathrm{~s}^{-1}\right]$

$P_{i} \quad$ partial pressure $[\mathrm{Pa}]$

$P_{t} \quad$ total pressure $[\mathrm{Pa}]$ 


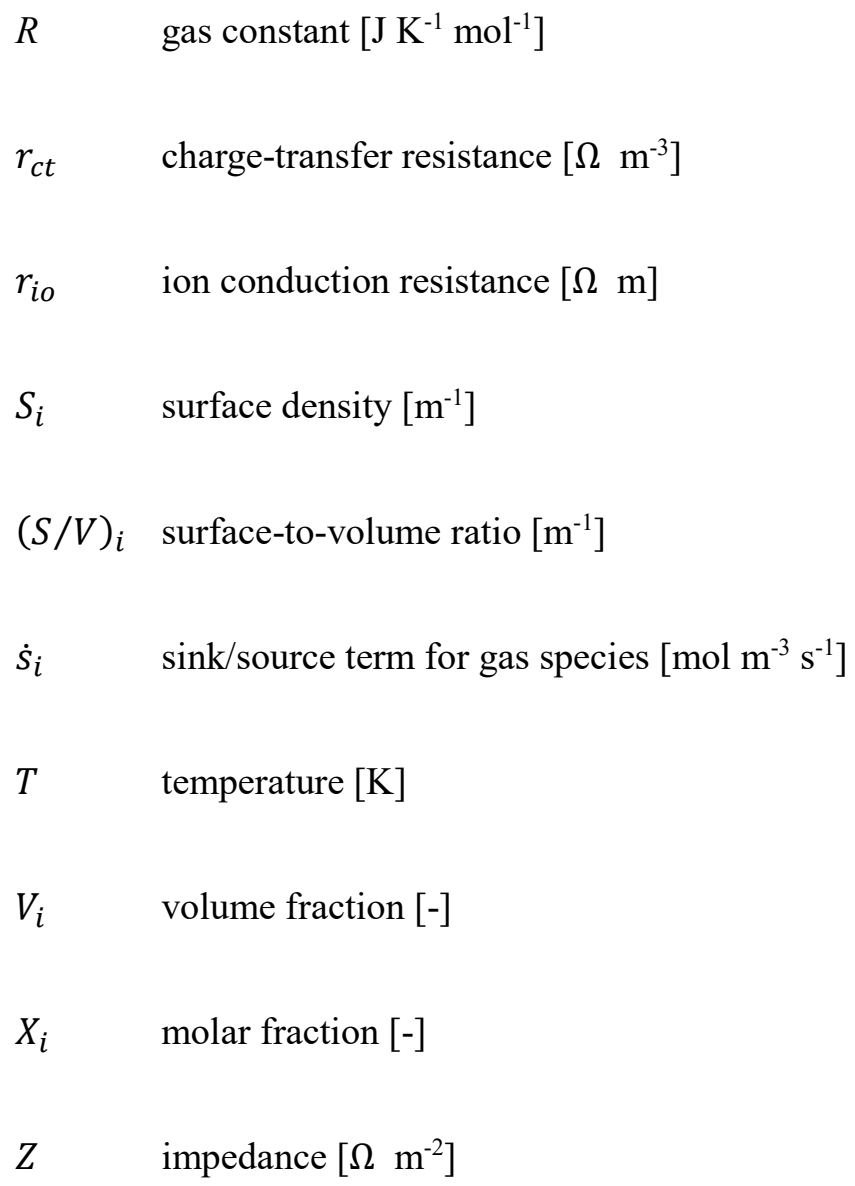

Greek symbols

$\eta_{\text {act }} \quad$ activation overpotential [V]

$\eta_{\text {con }} \quad$ concentration overpotential [V]

$\eta_{\mathrm{s}} \quad$ overpotential at the steady state $[\mathrm{V}]$

$\eta_{\mathrm{p}} \quad$ amplitude of the sinusoidal perturbation [V]

$\theta \quad$ period of the sinusoidal perturbation [s]

$\mu \quad$ viscosity [Pa s]

$\sum v_{i} \quad$ diffusion volume $\left[\mathrm{m}^{3}\right]$ 
$\sigma_{e l} \quad$ electronic conductivity [S m $\left.{ }^{-1}\right]$

$\sigma_{i o} \quad$ ionic conductivity [ $\left.\mathrm{S} \mathrm{m}^{-1}\right]$

$\tau_{i} \quad$ tortuosity factor [-]

$\phi_{i} \quad$ electric potential [V]

$\omega \quad$ angular velocity $\left[\mathrm{s}^{-1}\right]$

\section{Superscripts}

eff effective 


\section{References}

[1] M. Kadowaki, Current Status of National SOFC Project in Japan, ECS Transactions, 68 (2015) 15-22. $10.1149 / 07801.0033$ ecst

[2] J. Lewis, Stationary fuel cells - Insights into commercialisation, International Journal of Hydrogen Energy, 39 (2014) 21896-21901. 10.1016/j.ijhydene.2014.05.177

[3] Q.-A. Huang, R. Hui, B. Wang, J. Zhang, A review of AC impedance modeling and validation in SOFC diagnosis, Electrochimica Acta, 52 (2007) 8144-8164. 10.1016/j.electacta.2007.05.071

[4] J. Bisquert, G. Garcia-Belmonte, F. Fabregat-Santiago, A. Compte, Anomalous transport effects in the impedance of porous film electrodes, Electrochemistry Communications, 1 (1999) 429-435. 10.1016/S13882481(99)00084-3

[5] S.B. Adler, J.A. Lane, B.C.H. Steele, Electrode Kinetics of Porous Mixed-Conducting Oxygen Electrodes, Journal of the Electrochemical Society, 143 (1996) 3554-3564. 10.1149/1.1837252

[6] B. Boukamp, Interpretation of the Gerischer impedance in solid state ionics, Solid State Ionics, 157 (2003) 29-33. 10.1016/s0167-2738(02)00185-6

[7] A. Bertei, G. Arcolini, J.P. Ouweltjes, Z. Wuillemin, P. Piccardo, C. Nicolella, Physically-Based Deconvolution of Impedance Spectra: Interpretation, Fitting and Validation of a Numerical Model for Lanthanum Strontium Cobalt Ferrite-Based Solid Oxide Fuel Cells, Electrochimica Acta, 208 (2016) 129-141. 10.1016/j.electacta.2016.04.181

[8] A. Bertei, E. Ruiz-Trejo, F. Tariq, V. Yufit, A. Atkinson, N.P. Brandon, Validation of a physically-based solid oxide fuel cell anode model combining 3D tomography and impedance spectroscopy, International Journal of Hydrogen Energy, 41 (2016) 22381-22393. 10.1016/j.ijhydene.2016.09.100

[9] W.G. Bessler, A new computational approach for SOFC impedance from detailed electrochemical reactiondiffusion models, Solid State Ionics, 176 (2005) 997-1011. 10.1016/j.ssi.2005.01.002

[10] W. Lai, S.M. Haile, Impedance Spectroscopy as a Tool for Chemical and Electrochemical Analysis of Mixed Conductors: A Case Study of Ceria, Journal of the American Ceramic Society, 88 (2005) 2979-2997. 10.1111/j.1551-2916.2005.00740.x

[11] J.E. Mortensen, M. Sogaard, T. Jacobsen, Analytical, 1-Dimensional Impedance Model of a Composite Solid Oxide Fuel Cell Cathode, Journal of the Electrochemical Society, 161 (2013) F161-F175. 10.1149/2.077401jes [12] M. Kishimoto, H. Iwai, M. Saito, H. Yoshida, Quantitative evaluation of solid oxide fuel cell porous anode microstructure based on focused ion beam and scanning electron microscope technique and prediction of anode overpotentials, Journal of Power Sources, 196 (2011) 4555-4563. 10.1016/j.jpowsour.2010.12.100

[13] N. Shikazono, D. Kanno, K. Matsuzaki, H. Teshima, S. Sumino, N. Kasagi, Numerical Assessment of SOFC Anode Polarization Based on Three-Dimensional Model Microstructure Reconstructed from FIB-SEM Images, Journal of The Electrochemical Society, 157 (2010) B665. 10.1149/1.3330568

[14] M. Kishimoto, H. Iwai, M. Saito, H. Yoshida, Three-Dimensional Simulation of SOFC Anode Polarization Characteristics Based on Sub-Grid Scale Modeling of Microstructure, Journal of the Electrochemical Society, 159 (2012) B315-B323. 10.1149/2.086203jes

[15] U. Anselmi-Tamburini, G. Chiodelli, M. Arimondi, F. Maglia, G. Spinolo, Z.A. Munir, Electrical properties 
of Ni/YSZ cermets obtained through combustion synthesis, Solid State Ionics, 110 (1998) 35-43. 10.1016/S0167-2738(98)00115-5

[16] E.N. Fuller, P.D. Schettler, J.C. Giddings, A new method for prediction of binary gas-phase diffusion coefficients, Industrial and Engineering Chemistry, 58 (1966) 19-27. 10.1021/ie50677a007

[17] M.C. Doppler, J. Fleig, M. Bram, A.K. Opitz, The Capacitance of Nickel Pattern Electrodes on Zirconia Electrolyte, Journal of the Electrochemical Society, 163 (2016) H1019-H1025. 10.1149/2.0951610jes

[18] T. Kawada, N. Sakai, H. Yokokawa, M. Dokiya, Characteristics of Slurry-Coated Nickel Zirconia Cermet Anodes for Solid Oxide Fuel Cells, Journal of the Electrochemical Society, 137 (1990) 3042-3047. $10.1149 / 1.2086156$

[19] M. Kishimoto, M. Lomberg, E. Ruiz-Trejo, N.P. Brandon, Enhanced triple-phase boundary density in infiltrated electrodes for solid oxide fuel cells demonstrated by high-resolution tomography, Journal of Power Sources, 266 (2014) 291-295. 10.1016/j.jpowsour.2014.05.038

[20] M. Kishimoto, M. Lomberg, E. Ruiz-Trejo, N.P. Brandon, Numerical modeling of nickel-infiltrated gadolinium-doped ceria electrodes reconstructed with focused ion beam tomography, Electrochimica Acta, 190 (2016) 178-185. 10.1016/j.electacta.2015.12.044

[21] V. Sonn, A. Leonide, E. Ivers-Tiffée, Combined Deconvolution and CNLS Fitting Approach Applied on the Impedance Response of Technical Ni/8YSZ Cermet Electrodes, Journal of The Electrochemical Society, 155 (2008) B675-B679. 10.1149/1.2908860

[22] N.F. Bessette II, W.J. Wepfer, J. Winnick, A Mathematical Model of a Solid Oxide Fuel Cell, Journal of the Electrochemical Society, 142 (1995) 3792-3800. 10.1149/1.2048415

[23] S. Gewies, W.G. Bessler, Physically Based Impedance Modeling of Ni/YSZ Cermet Anodes, Journal of The Electrochemical Society, 155 (2008) B937. 10.1149/1.2943411

[24] B. de Boer, SOFC Anode: Hydrogen oxydation at porous nickel and nickel/yttria-stabilised zirconia cermet electrodes, Ph.D. Thesis, University of Twente, The Netherland, (1998).

[25] A. Bieberle, L.P. Meier, L.J. Gauckler, The Electrochemistry of Ni Pattern Anodes Used as Solid Oxide Fuel Cell Model Electrodes, Journal of The Electrochemical Society, 148 (2001) A646. 10.1149/1.1372219

[26] E.-C. Shin, J. Ma, P.-A. Ahn, H.-H. Seo, D.T. Nguyen, J.S. Lee, Deconvolution of Four Transmission-LineModel Impedances in Ni-YSZ/YSZ/LSM Solid Oxide Cells and Mechanistic Insights, Electrochimica Acta, 188 (2016) 240-253. 10.1016/j.electacta.2015.11.118

[27] R. Mohammadi, M. Søgaard, T. Ramos, M. Ghassemi, M.B. Mogensen, Electrochemical Impedance Modeling of a Solid Oxide Fuel Cell Anode, Fuel Cells, 14 (2014) 645-659. 10.1002/fuce.201300292 